\title{
Improved functionalization of oleic acid-coated iron oxide nanoparticles for biomedical applications
}

\author{
Maarten Bloemen - Ward Brullot • \\ Tai Thien Luong • Nick Geukens • Ann Gils • \\ Thierry Verbiest
}

Received: 27 February 2012/Accepted: 24 July 2012/Published online: 7 August 2012

(C) The Author(s) 2012. This article is published with open access at Springerlink.com

\begin{abstract}
Superparamagnetic iron oxide nanoparticles can provide multiple benefits for biomedical applications in aqueous environments such as magnetic separation or magnetic resonance imaging. To increase the colloidal stability and allow subsequent reactions, the introduction of hydrophilic functional groups onto the particles' surface is essential. During this process, the original coating is exchanged by preferably covalently bonded ligands such as trialkoxysilanes. The duration of the silane exchange reaction, which commonly takes more than $24 \mathrm{~h}$, is an important drawback for this approach. In this paper, we present a novel method, which introduces ultrasonication as an energy source to dramatically accelerate this process, resulting in high-quality waterdispersible nanoparticles around $10 \mathrm{~nm}$ in size. To prove
\end{abstract}

Electronic supplementary material The online version of this article (doi:10.1007/s11051-012-1100-5) contains supplementary material, which is available to authorized users.

M. Bloemen $(\varangle) \cdot$ W. Brullot · T. T. Luong · T. Verbiest Department of Chemistry, KU Leuven, Celestijnenlaan 200D, Box 2425, 3001 Heverlee (Leuven), Belgium e-mail: maarten.bloemen@fys.kuleuven.be

N. Geukens

PharmAbs, The KU Leuven Antibody Center,

KU Leuven, O\&N II, Herestraat 49,

Box 824, 3000 Leuven, Belgium

A. Gils

Faculty of Pharmaceutical Sciences, KU Leuven, O\&N II Herestraat 49, Box 824, 3000 Leuven, Belgium the generic character, different functional groups were introduced on the surface including polyethylene glycol chains, carboxylic acid, amine, and thiol groups. Their colloidal stability in various aqueous buffer solutions as well as human plasma and serum was investigated to allow implementation in biomedical and sensing applications.

Keywords Oleic acid-coated nanoparticles . Superparamagnetic $\cdot$ Surface modification - Silane Colloidal stability · Iron oxide

\section{Introduction}

For many years, iron oxide nanoparticles have been the subject of intensive research. These cost-effective and non-toxic particles are used nowadays in many applications such as magnetic storage, drug delivery, biosensing, magnetic separation, and contrast reagents for imaging techniques (Laurent et al. 2008; Speliotis 1999). A common method to make such particles is coprecipitation where iron(II+) and (III+) ions are dissolved in water and precipitated using ammonia or sodium hydroxide (Sun et al. 2006). Drawbacks are the poor monodispersity and irregular shape of these particles. Recently, multiple methods have been published to synthesize monodisperse nanoparticles in organic solvents. Sun et al. (2004) made high-quality magnetite nanocrystals with small size distribution by thermal decomposition of iron(III) acetylacetonate in 
phenyl ether. A similar method reported by Park et al. (2004) uses iron-oleate as a precursor and oleic acid as a capping agent. This results in nanoparticles with a hydrophobic coating since the polar end groups are attached to the surface. Capping agents such as oleic acid are often used because they form a protective monolayer, which is strongly bonded. This is necessary for making monodisperse and highly uniform nanoparticles (Bronstein et al. 2007; Zhang et al. 2006). For biomedical applications in aqueous environments, this hydrophobic coating has to be replaced with a hydrophilic coating. The so-called ligand exchange is well known for noble metal nanoparticles where, for instance, thiol groups attach strongly to the surface, thereby forming monolayers by self-assembly. A similar approach is possible for iron oxide nanoparticles using polymers or $\alpha$-cyclodextrin (Fauconnier et al. 1997; Wang et al. 2003; Park et al. 2011). Since these layers are often not covalently bonded to the surface, high ionic strength or extreme $\mathrm{pH}$ conditions might alter their interaction. An elegant alternative is silane chemistry, which is based on the reactivity of silanol molecules, formed by the hydrolyzation of alkoxy silane (Soderholm and Shang 1993; Zhang et al. 2002). A high degree of control and reproducibility is possible when the appropriate reaction conditions are chosen. The possibility to introduce a large variety of functional groups onto the surface of magnetic nanoparticles makes this approach very valuable. Other advantages are the high stability and density of the formed silicon oxide layer. However, introducing silane molecules onto the surface of oleic acidstabilized nanoparticles has only been scarcely reported so far. De Palma et al. (2007) described a method which uses hexane as a solvent and acetic acid as a catalyst to form the reactive silanol molecules. Larsen et al. (2009) published a protocol with toluene as the solvent and water being the catalyst; triethylamine was added to facilitate the reaction. Kohler et al. (2004) also managed to introduce silanes, but pretreated the oleic acid coating with a mixture of $1 \mathrm{M}$ ammonium hydroxide in 1-butanol. These methods have serious drawbacks, e.g., the extensive reaction time (24-72 h) or pre-treatment procedure.

In this paper, we present an improved nanoparticles functionalization method. The obtained superparamagnetic nanoparticles are thoroughly characterized by electron microscopy, X-ray powder diffraction, and vibrating sample magnetometry. Infrared spectroscopy is used to confirm the presence of the functional groups on the surface after reaction with silane molecules. By performing this reaction in an ultrasonication bath, the reaction time is greatly reduced, while avoiding crosslinking and thus maintaining the monodispersity. The colloidal stability of the resulting nanoparticles was extensively tested in different aqueous media at several $\mathrm{pHs}$ as well as in human serum and plasma, which demonstrates their applicability in biomedical applications. A large variety of functional groups were introduced to the surface, proving the generic character of the method.

\section{Materials and methods}

\section{Materials}

Sodium oleate and iron(III) chloride hexahydrate (97\%) were obtained from Sigma Aldrich, ethanol (absolute) and oleic acid from VWR, and heptane and toluene from Fisher Scientific. Triethyl amine was ordered at Janssen Chimica. Acetone was purchased at Chem Lab. Methoxy(polyethyleneoxy)propyltrimethoxysilane (90\%, 9-12 PE-units), 3-mercaptopropyltrimethoxysilane (95\%), $\mathrm{N}$-(trimethoxysilylpropyl) ethylenediamine triacetic acid trisodium salt (45\%), and 3-Aminopropyltrimethoxysilane (97\%) were obtained from ABCR. 1-Octadecene (90\%, technical grade) was purchased at Acros.

Synthesis of nanoparticles

Superparamagnetic iron oxide nanoparticles were prepared by the method published by Park et al. (2004) with minor modifications. It consists of two separate reactions, first preparing an iron-oleate precursor, which is later transformed into iron oxide nanocrystals.

For the synthesis of the precursor, $36.5 \mathrm{~g}(120 \mathrm{mmol})$ sodium oleate and $10.8 \mathrm{~g}$ (40 mmol) iron(III) chloride hexahydrate were dissolved in a mixture of $80 \mathrm{~mL}$ ethanol, $60 \mathrm{~mL}$ MilliQ water, and $140 \mathrm{~mL}$ heptane. This mixture was heated to reflux at $70{ }^{\circ} \mathrm{C}$ for $4 \mathrm{~h}$ under an argon atmosphere. Afterward, the upper heptane layer, which contains the iron-oleate, was separated using a separatory funnel and washed three times with $40 \mathrm{~mL}$ MilliQ water. As a final step, the heptane was evaporated using a rotavapor, resulting in a dark brown waxy solid. 
The iron oxide nanoparticles' synthesis starts with

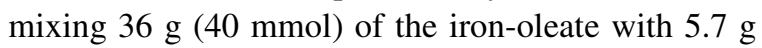
(20 mmol) of oleic acid and $200 \mathrm{~g}$ of 1-octadecene in a $500 \mathrm{~mL}$ three-neck flask. This mixture was first heated to $100{ }^{\circ} \mathrm{C}$ for $5 \mathrm{~min}$ to evaporate all remaining heptane. After fitting a reflux cooler, the mixture was heated further to $320{ }^{\circ} \mathrm{C}$ and kept at that temperature for $30 \mathrm{~min}$. Around $250{ }^{\circ} \mathrm{C}$, the decarboxylation of the oleate starts, producing a large amount of $\mathrm{CO}_{2}$ gas. Afterward, the reaction mixture is cooled down to room temperature by removing the heat source. $500 \mathrm{~mL}$ of ethanol is added to precipitate the freshly prepared nanoparticles. Separation was done by centrifugation, after which the particles were washed three times with ethanol. After drying, the nanocrystals were dispersed in heptane (with one drop of oleic acid) in high concentration $(100 \mathrm{mg} / \mathrm{mL})$ for longterm storage.

\section{Functionalization}

The new protocol presented here was partially based on a method published by Larsen et al. (2009), but with important modifications. In a typical functionalization experiment, $100 \mathrm{mg}$ of iron oxide nanoparticles (in heptane, stock solution) were mixed with $50 \mathrm{~mL}$ of toluene. To this mixture, $2.5 \mathrm{~mL}$ of triethylamine, $0.05 \mathrm{~mL}$ of MilliQ water, and $0.5 \mathrm{~mL}$ of the desired silane were added. The beaker was then placed in an ultrasonication bath for $5 \mathrm{~h}$. The temperature of the water inside this bath was kept at $50{ }^{\circ} \mathrm{C}$ during the reaction. Afterward, the volume of the reaction mixture was doubled by adding heptane to the solution, $50 \mathrm{~mL}$ in this case. The mixture was placed on a magnet to precipitate the functionalized nanoparticles. The supernatant was decanted and the particles were washed three times with acetone and precipitated by a magnet. After drying under reduced pressure for $15 \mathrm{~min}$, the sample was weighed and dissolved in MilliQ water or the appropriate medium.

\section{Equipment and characterization}

The ultrasonication bath used in the particle functionalization was a Bransonic Model 5510 sonicator with a capacity of $10 \mathrm{~L}$. The built-in heating was never used.

Transmission electron microscopy measurements were performed on a $80 \mathrm{kV}$ Zeiss EM-900 using 300 mesh Formvar coated copper grids. Distribution data were calculated by ImageJ. Oleic acid-coated nanoparticles were dispersed in heptane and deposited onto the grid.

Fourier transform infrared spectra were obtained using a Bruker Alpha FT-IR spectrometer equipped with a Platinum ATR module.

Dynamic light scattering and zeta potentials were measured on a Brookhaven 90plus particle analyzer. The internal detector was positioned at $90^{\circ}$.

UV-Vis Spectrometry was performed on a Perkin Elmer Lambda 900 spectrometer.

Vibrating sample magnetometry experiments were conducted on a VSM Maglab setup from Oxford Instruments.

X-ray powder diffraction spectra were obtained in reflection (Bragg-Brentano geometry) using a Rigaku Rotaflex diffractometer fitted with a Rigaku RU-200B rotating $\mathrm{Cu}$-anode $(\lambda=1.54 \AA$ ) at a power of $4 \mathrm{~kW}$. The diffracted $X$-rays were collected after Ni-filtering on a scintillation counter. Samples were deposited on a glass microscope slide from solution.

Samples, to test the colloidal stability, were prepared by adding concentrated nanoparticle dispersion (in water) to the appropriate medium. The protocol for collection of human plasma and serum is described in Online Resource 1. Absorbance values were measured at a wavelength of 1,000 $\mathrm{nm}$.

\section{Results and discussion}

Efficient surface modification of superparamagnetic nanoparticles is crucial for their application in biomedicine. Recent advances in high temperature syntheses already improved the shape and monodispersity of the core. As mentioned before, these particles are only soluble in apolar solvents because of their oleic acid coating. To change the polarity of the layer to being hydrophilic, a ligand exchange is essential. We preferred interaction with alkoxy silanes to polymers because of the covalent bond formation, resulting in more versatile and robust nanoparticles. The reaction mechanism is shown in Fig. 1. After formation of the active silanol molecule, it reacts with the surface $\mathrm{OH}$ groups of the iron oxide nanoparticle. This results in the formation of a $\mathrm{Fe}-\mathrm{O}-\mathrm{Si}$ bond. Metal oxides are known to have reactive hydroxyl groups present on their surface, caused by the adsorption of water (Cornell and Schwertmann 2003). This is similar to 
Fig. 1 Overview of the chemical reactions during the functionalization of iron oxide nanoparticles with silanes. The formation of the silanol molecule occurs by reaction with water.

Subsequent

polycondensation renders a

silane network on the surface of the nanoparticle
Oleic acid-coated iron oxide
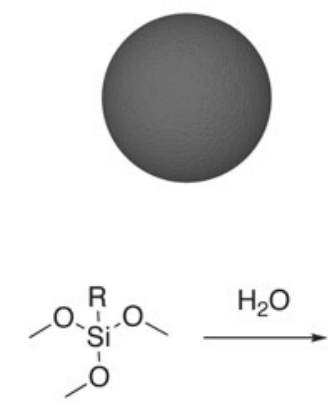

Trialkoxy silane

functionalization of silicon oxide substrates (Fadeev and McCarthy 2000). The density and structure of the shell depends largely on the reaction conditions, being a combination of linear polymerization, network formation, and covalent attachment to the iron oxide surface. Although chlorosilanes would react faster than alkoxy silanes, we opted for the latter. Chlorosilanes release hydrogen chloride upon reaction, which is incompatible with iron oxide. On top of that, their reactivity is often too high to allow sufficient control of the reaction.

The nanoparticle synthesis published by Park et al. (2004) allowed us to make high-quality spherical nanoparticles at large scale. Figure 2 shows a transmission electron microscopy (TEM) image of the oleic acid-coated nanoparticles. As can be seen in the inset, the nanoparticle distribution is narrow. A mean size of $9.3 \mathrm{~nm}$ was calculated by a Gaussian fit with a spread of $\pm 1.6 \mathrm{~nm}$ (one sigma).

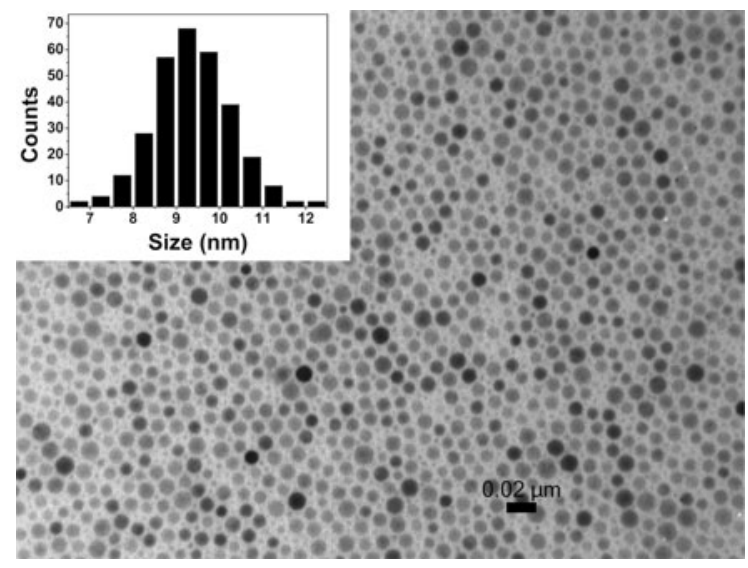

Fig. 2 Transmission electron microscopy image of oleic acidstabilized iron oxide nanoparticles. The scale bar represents $20 \mathrm{~nm}$. The inset shows the size distribution: $9.3 \pm 1.6 \mathrm{~nm}$

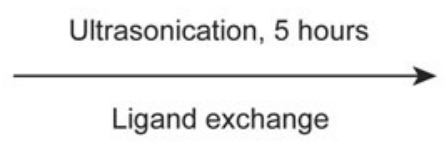

Functionalized shell
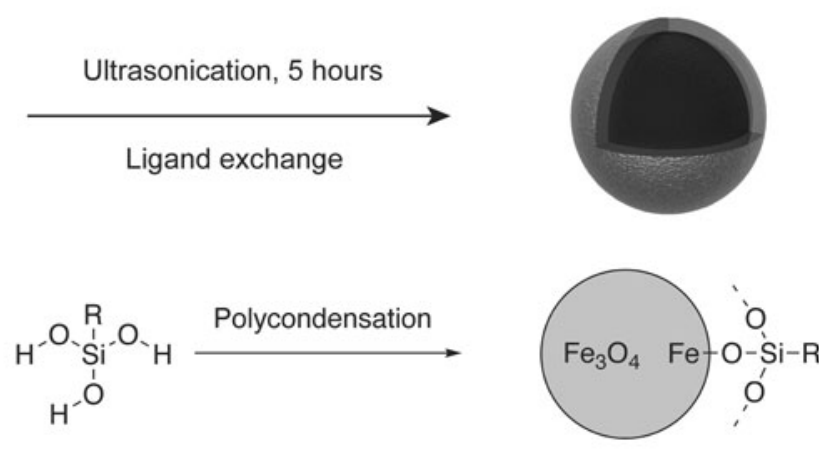

Silanol

Since superparamagnetism is an important property of the nanoparticles, vibrating sample magnetometry (VSM) measurements were performed. Figure 3 shows the data of the oleic acid-coated particles. The applied field was varied between 4 and -4 Tesla, while recording the remnant magnetization. No coercivity or magnetic remanence is observed, which is typical for superparamagnetic nanoparticles. The hysteresis loop can be fitted by a Langevin function to deduct the size of the magnetic core. In this case, a core diameter of $10.53 \mathrm{~nm}$ was obtained. This value is comparable to the size determined by TEM measurements.

The crystal structure of the oleic acid-coated iron oxide nanoparticles was determined via $\mathrm{X}$-ray powder diffraction ( step size $=0.05^{\circ}$, dwell time $=40 \mathrm{~s}$ ). As Fig. 4 indicates, the spectrum closely resembles the reference spectrum of magnetite. However, since the difference between maghemite and magnetite is very subtle in X-ray diffraction, no conclusions about the exact composition can be drawn. Therefore, we can only state that the crystals produced in the synthesis are superparamagnetic iron oxide nanoparticles, consisting of magnetite, maghemite, or a mixture of both. By means of the Scherrer equation, the crystal size can be derived from the peak broadening in the spectrum (Brullot et al. 2012). By means of MDI Jade, the (400) peak was fitted with a pseudo-Voight function after polynomial background subtraction, resulting in a size of $9.3 \pm 0.7 \mathrm{~nm}$. This value corresponds very well with the size determined by the TEM measurements.

Compared to previously reported functionalization methods, the method presented here has several advantages. (1) The reaction time is reduced to $5 \mathrm{~h}$ compared to 24 or $72 \mathrm{~h}$ as described by Larsen et al. (2009) and De Palma et al. (2007). (2) Using a sonicator, crosslinking of particles during the reaction 


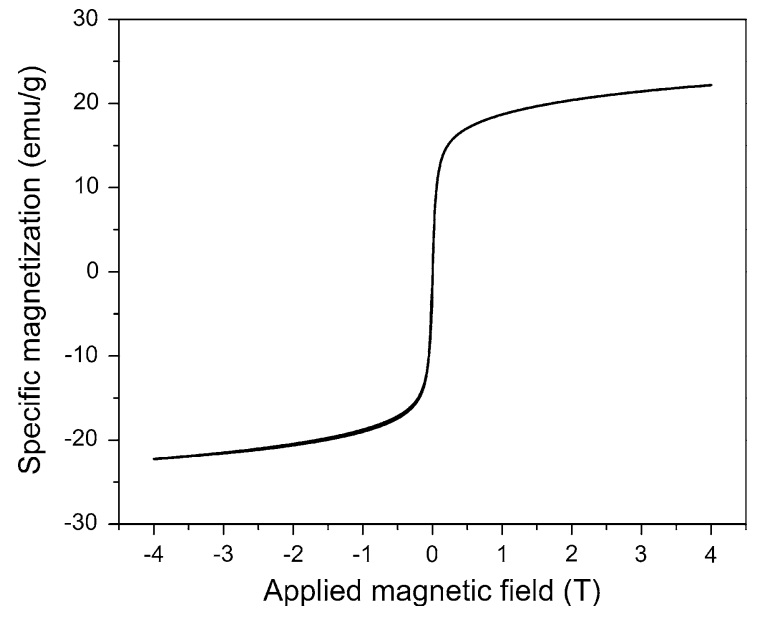

Fig. 3 Vibrating sample magnetometry signal of the nanoparticles showing a hysteresis curve. The data points are fitted by a Langevin function to determine the magnetic core size

is strongly reduced, thereby maintaining the monodispersity of the nanoparticles. In this case, the exact mechanism is unknown, but in general, sonication of a solution introduces microbubbles, which subsequently implode. These implosions generate high temperatures inside and around the cavity as well as a shock wave upon collapse. The surrounding liquid quickly disperses the heat, allowing the use of fragile organic materials (Mason and Lorimer 2002; Morel et al. 2008). (3) Another advantage is the elevated

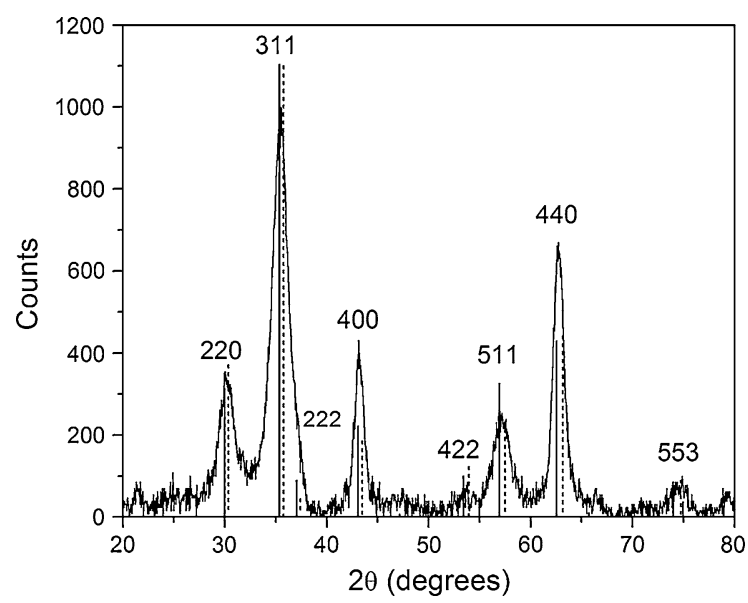

Fig. 4 X-ray powder diffraction spectrum of the oleic acidcoated iron oxide nanoparticles. The solid and dashed vertical drop-down lines represent the peak positions of a reference magnetite and maghemite spectrum, respectively (AMCSD 0007824 and 0007899) concentration of nanoparticles $(2 \mathrm{mg} / \mathrm{mL})$ during the synthesis, which reduces the amount of solvent needed.

The functionalization procedure was performed with four different trialkoxy silanes. Polyethylene glycol (PEG), carboxylic acid, amine, and thiol groups were introduced on the surface for various reasons. Thiol and amine groups are excellent anchor points for subsequent coating with a gold layer, e.g., by reduction of a gold containing salt. This is particularly useful in biomedical applications where the plasmonic response of gold is used to heat the environment or to release drugs at a specific location (Brullot et al. 2011; Janib et al. 2010). PEG chains on the surface, on the other hand, provide the nanoparticles with excellent dispersibility in water. These particles can be used to form magnetic fluids for applications like magnetic hyperthermia or thermo-ablation (Prasad et al. 2007). The introduction of carboxylic acid and amine groups can be utilized for bioconjugation of proteins to the nanoparticles. Typical chemical coupling reagents like EDC-NHS or glutaraldehyde link these functional groups to the target protein via an amide or imine bond. These bioconjugates are often used for detection and magnetic separation.

Successful modification of the nanoparticles' surface was confirmed by Fourier transform infrared measurements (FTIR). Table 1 gives an overview of the vibrations measured on the oleic acid-and silane-coated particles. For the unmodified iron oxide cores, the characteristic bands of the asymmetric stretching, symmetric stretching, and scissoring of $\mathrm{CH}_{2}$ are visible at 2,919,2,850, and $1,436 \mathrm{~cm}^{-1}$, respectively (Zhang et al. 2006; Chen et al. 2008a, b). The stretching of the $\mathrm{C}=\mathrm{O}$ double bond is clearly shown by the peak at $1,709 \mathrm{~cm}^{-1}$. This was expected since the peak resembles free oleic acid, indicating that a significant amount of unbound surfactant is present, which can be related to the storage conditions. The presence of a peak at $1,462 \mathrm{~cm}^{-1}$, coming from in-plane $\mathrm{OH}$ bending, supports this idea. Zhang et al. (2006) reported that the $\mathrm{C}=\mathrm{O}$ peak shifts to 1,541 and $1,639 \mathrm{~cm}^{-1}$ when the molecule is attached to a ferrite surface. The carboxylic acid groups are then present in a $\mathrm{COO}^{-}$conformation. If the spectrum is magnified, these vibrations are also visible in our case at 1,541 and $1,635 \mathrm{~cm}^{-1}$, although they are fairly small. The wavenumber separation (of $94 \mathrm{~cm}^{-1}$ ) between those two peaks is an indication for how the oleate and the iron atoms on the surface interact. 
Table 1 Overview of the different vibrations related to the different coatings around the nanoparticle

\begin{tabular}{|c|c|}
\hline $\begin{array}{l}\text { Surface of } \\
\mathrm{Fe}_{3} \mathrm{O}_{4}\end{array}$ & IR vibrations $\left(\mathrm{cm}^{-1}\right)$ \\
\hline Oleic acid & $\begin{array}{l}\text { 3,005 (HC=), 2,919 }\left(\mathrm{CH}_{2}\right), 2,850\left(\mathrm{CH}_{2}\right), 1,709(\mathrm{C}=0), 1,635\left(\mathrm{COO}^{-}\right), 1,541\left(\mathrm{COO}^{-}\right), 1,462(\mathrm{OH}), 1,436\left(\mathrm{CH}_{2}\right), 598 \\
(\mathrm{Fe}-\mathrm{O})\end{array}$ \\
\hline $\mathrm{COOH}$ silane & $\begin{array}{l}3,600-3,000(\mathrm{OH}), 2,932\left(\mathrm{CH}_{2}\right), 1,612\left(\mathrm{COO}^{-}\right), 1,452\left(\mathrm{CH}_{2}\right), 1,396\left(\mathrm{COO}^{-}\right), 1,113 \& 1,089 \& 1,007(\mathrm{Si}-\mathrm{O}), 585 \\
(\mathrm{Fe}-\mathrm{O})\end{array}$ \\
\hline PEG silane & $\begin{array}{l}\left.\text { 3,600-3,000 (OH), 2,860 ( } \mathrm{PEG} \mathrm{CH}_{2}\right), 1,643\left(\mathrm{H}_{2} \mathrm{O}\right), 1,454 \& 1,349 \& 1,297 \& 1,250 \& 1,047 \& 947\left(\mathrm{CH}_{2}-\mathrm{O}-\mathrm{CH}_{2}\right), \\
\text { 1,198 (O-CH3), } 620(\mathrm{Fe}-\mathrm{O})\end{array}$ \\
\hline $\mathrm{NH}_{2}$ silane & $\begin{array}{l}\left.\text { 3,004 (OH \& } \mathrm{NH}_{2}\right), 2,922\left(\mathrm{CH}_{2}\right), 2,850\left(\mathrm{CH}_{3}\right), 1,543\left(\mathrm{NH}_{3}^{+}\right), 1,400\left(\mathrm{CH}_{3} \mathrm{COOH}\right), 1,224(\mathrm{Si}-\mathrm{C}), 1,073(\mathrm{Si}-\mathrm{O}-\mathrm{R}), \\
773\left(\mathrm{NH}_{2}\right), 617(\mathrm{Fe}-\mathrm{O})\end{array}$ \\
\hline SH silane & 3,600-2,500 ( $\left.\mathrm{OH} \& \mathrm{CH}_{2}\right), 2,600-2,550(\mathrm{SH}), 1,645\left(\mathrm{H}_{2} \mathrm{O}\right), 1,430\left(\mathrm{CH}_{2}\right), 1,035(\mathrm{Si}-\mathrm{O}), 590(\mathrm{Fe}-\mathrm{O})$ \\
\hline
\end{tabular}

The original spectra can be found in Online Resource 1

Because the difference is smaller than $110 \mathrm{~cm}^{-1}$, a chelating bidentate interaction can be derived from the spectrum. The iron oxide core itself shows a characteristic vibration at $598 \mathrm{~cm}^{-1}$, related to the $\mathrm{Fe}-\mathrm{O}$ bonds (Zhang et al. 2006).

The presence of carboxylic acid groups, after functionalization, is proven by the vibrations at 1,612 and $1,396 \mathrm{~cm}^{-1}$. These correspond to the asymmetric and symmetric stretching of the $\mathrm{COO}^{-}$group, respectively. Other important features are the 1,113, 1,089, and $1,007 \mathrm{~cm}^{-1}$ bands due to the stretching of the $\mathrm{Si}-\mathrm{O}$ bond (Can et al. 2009; Ma et al. 2003).

The PEG silane shows distinct bands caused by the ether functions in the chain, to which several peaks between 1,454 and $947 \mathrm{~cm}^{-1}$ can be attributed. The high hydrophilicity of the PEG chain is expressed by the presence of a water peak in the spectrum. Even after extensive drying, this peak remains, indicating that the water is trapped by hydrogen bonding (De Palma et al. 2007). Several papers already reported that more information about the structure of the PEG layer on the nanoparticles surface can be derived from the FTIR spectrum. Compared to these reports, the conformation of the poly ethylene glycol layer is partially crystalline and partially amorphous. Small shoulders at $1,244,1,460$, and $1,470 \mathrm{~cm}^{-1}$ are present in the spectrum, indicating crystalline parts in the coating. On the other hand, the amorphous bands are visible at 948, 1,140, and $1,250 \mathrm{~cm}^{-1}$ (Harder et al. 1998; Valiokas et al. 1999).

For the amino silane-coated nanoparticles, the most important peaks are visible at 3,004, 1,543, and $773 \mathrm{~cm}^{-1}$ corresponding to the protonated amines. Because this protonation was done by adding acetic acid, a peak at $1,400 \mathrm{~cm}^{-1}$ appears. Mercapto silane- coated particles show a broad band above $2,500 \mathrm{~cm}^{-1}$, consisting of a combination of $\mathrm{OH}, \mathrm{CH}_{2}$, and $\mathrm{SH}$ vibrations. Also, a small peak caused by the presence of water is visible. Typical peaks for $\mathrm{Si}-\mathrm{O}$ en $\mathrm{Fe}-\mathrm{O}$ also appear at 1,035 and $590 \mathrm{~cm}^{-1}$, respectively.

Further experiments were only conducted on the PEG, $\mathrm{COOH}$, and amine coatings, since the thiol particles were only stable in water for a very short time, regardless of the $\mathrm{pH}$. In general, the stability of nanoparticles dispersed in aqueous media can be expressed by the zeta potential. Theoretically, it refers to the potential difference between the slipping plane in the electronic double layer and the bulk potential. If the potential has an absolute value higher than $25-30 \mathrm{mV}$, it is generally accepted that the particles are electrostatically stable (Xu 2001). Although colloidal stability is related to electrostatic and steric repulsion, zeta potential measurements usually give a good indication. Figure 5 shows the combined data of the PEG-, carboxylic acid-, and amine-coated nanoparticles. A clear downward trend for the zeta potential of $\mathrm{NH}_{2}$ is visible when the $\mathrm{pH}$ increases. This can be related to the lowering of the surface charge due to deprotonation of the amine at high $\mathrm{pH}$. A similar, but reverse, trend can be observed for $\mathrm{COOH}$ since the acid becomes protonated at low $\mathrm{pH}$, thereby losing its negative charge. For PEG-coated particles, the zeta potential shows similar behavior even though polyethylene glycol chains have no $\mathrm{pH}$ responsive groups. The incorporation of ions into the PEG layer can explain this trend, taking into consideration that sodium hydroxide and hydrogen chloride were added to adjust the $\mathrm{pH}$ of the solution (Schweiss et al. 2001; Kreuzer et al. 2003). Nevertheless, the PEG-coated particles were stable over the entire $\mathrm{pH}$ range, 


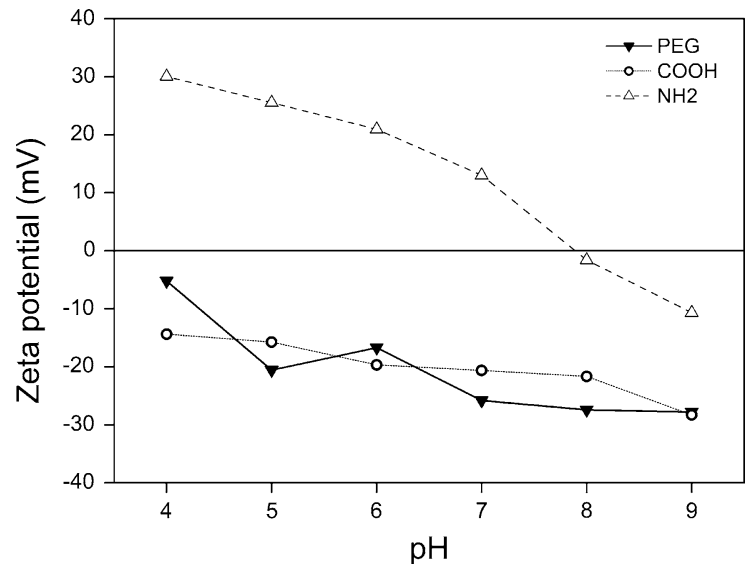

Fig. 5 Zeta potential values for PEG-, carboxylic acid-, and amine-coated nanoparticles in various $\mathrm{pH}$ solutions. Every data point was derived from 10 measurements by the software

indicating that their stability is caused by steric repulsion rather than electrostatic repulsion. For the $\mathrm{COOH}$-coated nanoparticles, solutions of $\mathrm{pH} 4$ and 5 showed extensive aggregation and consequent precipitation. A similar effect was observed for the aminecoated particles, this time for dispersions of $\mathrm{pH} 7$ to 9 . The profound impact of the $\mathrm{pH}$ on the stability of $\mathrm{COOH}$ and $\mathrm{NH}_{2}$ coatings shows that electrostatic repulsion is crucial for these types of dispersions (De Palma et al. 2007; Chen et al. 2008a, b).

To study the effect of buffer solutions on the colloidal stability, various dispersions with different salt concentrations were prepared. To our knowledge, no extensive studies have been conducted on magnetic nanoparticle dispersions in different buffer media at different pHs and concentrations (Barreto et al. 2011; Bhattacharya et al. 2011). Nevertheless, this information can be very valuable for subsequent reactions or applications of the nanoparticles. Five commonly used buffer reagents were chosen, covering the entire $\mathrm{pH}$ range between 4 and 9 . These included sodium acetate, 2-( $N$-morpholino)-ethanesulfonic acid (MES), sodium hydrogen phosphate, 2-amino-2-hydroxymethyl-propane-1,3-diol (TRIS), and glycine. The concentration of the reagents ranged from 0.1 to $0.025 \mathrm{M}$, while the particle concentration was fixed at $0.25 \mathrm{mg} / \mathrm{mL}$. Table 2 provides an overview of the stability in different buffer solutions, each having three different concentrations. Carboxylic acid-coated nanoparticles show excellent stability in different buffers above $\mathrm{pH}$ 4 , which can be related to the presence of charged carboxylate groups on their surface, providing sufficient electrostatic repulsion. For the $\mathrm{NH}_{2}$-coated particles, the instability can be explained by the lack of charged functional groups. This is partially caused by the $\mathrm{pH}$, which is too high for sufficient stabilization. On top of that, the high ionic strength and ion size largely influence the zeta potential. These results are in perfect agreement with the zeta potential measurements and the observed correlation between the presence of surface charges and colloidal stability. The particles with polyethylene glycol chains on their surface show good stability in phosphate, glycine, and TRIS-based buffer solutions. The instability in MES and acetate buffer, however, is somewhat unexpected since the zeta potential measurements showed that PEG provides mainly steric hindrance rather than electrostatic repulsion. Ion adsorption onto the coated surface is expected to be the cause of this instability.

If superparamagnetic nanoparticles are used as, for example, an MRI contrast reagent, colloidal stability in (human) serum or plasma is crucial. To prove the value of the presented functionalization method, nanoparticle dispersions in both serum and plasma were prepared and their stability was monitored in time by absorption measurements (see Online Resource 1). Human blood has a $\mathrm{pH}$ of 7.4, which will strongly influence the stability of the nanoparticles as was shown in previous paragraphs. Similarly, for the dispersions in buffer solutions, three different coatings were tested, amine, carboxylic acid, and PEG functional groups. These nanoparticles were dispersed in serum and plasma at a concentration of 1 and $0.25 \mathrm{mg} / \mathrm{mL}$. In accordance with the previous results, amine groups on the surface cannot provide sufficient electrostatic repulsion at $\mathrm{pH} 7.4$, which resulted in a rapid decline of the absorbance. On the contrary, carboxylic acid groups and PEG chains should be able to provide sufficient stabilization in these conditions. This was observed in both cases since the absorbance remained constant during the entire experiment, which lasted $48 \mathrm{~h}$. These results indicate that the PEG and carboxylic acid-coated nanoparticles can be of great importance for future in vivo experiments.

An important remark about the use of nanoparticles in biomedical applications is their possible toxicity toward cells. Although many conflicting results were published about the toxicity of superparamagnetic iron oxide nanoparticles, a study by Mahmoudi et al. (2011) recently showed that the surface coating and the cell type itself have a large influence on the 
Table 2 Overview of the stability of the nanoparticles in buffer solutions

\begin{tabular}{|c|c|c|c|c|c|c|c|c|c|}
\hline \multirow{3}{*}{$\begin{array}{l}\text { Coating } \\
100 \%\end{array}$} & \multicolumn{3}{|c|}{$\mathrm{CH}_{3} \mathrm{COOH} / \mathrm{CH}_{3} \mathrm{COONa}$} & \multicolumn{3}{|c|}{$\mathrm{CH}_{3} \mathrm{COOH} / \mathrm{CH}_{3} \mathrm{COONa}$} & \multicolumn{3}{|c|}{$\mathrm{MES} / \mathrm{HCl}$} \\
\hline & \multicolumn{3}{|l|}{$\mathrm{pH} 4$} & \multicolumn{3}{|l|}{ pH5 } & \multicolumn{3}{|l|}{ pH6 } \\
\hline & $0.1 \mathrm{M}$ & $0.05 \mathrm{M}$ & $0.025 \mathrm{M}$ & $0.1 \mathrm{M}$ & $0.05 \mathrm{M}$ & $0.025 \mathrm{M}$ & $0.1 \mathrm{M}$ & $0.05 \mathrm{M}$ & $0.025 \mathrm{M}$ \\
\hline $\mathrm{NH}_{2}$ & + & + & + & + & + & + & \pm & \pm & + \\
\hline PEG & + & + & + & \pm & \pm & \pm & \pm & \pm & \pm \\
\hline $\mathrm{COOH}$ & \pm & + & + & + & + & + & + & + & + \\
\hline \multirow{3}{*}{$\begin{array}{l}\text { Coating } \\
100 \%\end{array}$} & \multicolumn{3}{|c|}{$\mathrm{NaH}_{2} \mathrm{PO}_{4} / \mathrm{Na}_{2} \mathrm{HPO} 4$} & \multicolumn{3}{|c|}{ TRIS/HCL } & \multicolumn{3}{|c|}{ Glycine/NaOH } \\
\hline & \multicolumn{3}{|l|}{$\mathrm{pH} 7$} & \multicolumn{3}{|l|}{ pH8 } & \multicolumn{3}{|l|}{ pH9 } \\
\hline & $0.1 \mathrm{M}$ & $0.05 \mathrm{M}$ & $0.025 \mathrm{M}$ & $0.1 \mathrm{M}$ & $0.05 \mathrm{M}$ & $0.025 \mathrm{M}$ & $0.1 \mathrm{M}$ & $0.05 \mathrm{M}$ & $0.025 \mathrm{M}$ \\
\hline $\mathrm{NH}_{2}$ & - & - & - & - & - & - & - & - & - \\
\hline PEG & + & + & + & + & + & + & + & + & + \\
\hline $\mathrm{COOH}$ & + & + & + & + & + & + & + & + & + \\
\hline
\end{tabular}

$(+)$ stands for excellent dispersibility and stability in time (minimum 1 week), $( \pm$ ) corresponds to colloidal solutions that are stable for $<4$ days, ( - stands for dispersions which are stable for only a short period of time (between 5 and $10 \mathrm{~h}$ ). The concentration of nanoparticles in solution was $0.25 \mathrm{mg} / \mathrm{mL}$. The criterion for stability was the absence of visible aggregation or precipitate

possible toxic effects (Zhang et al. 2002). They state that the introduction of a functional surface coating lowers the inherent toxicity. Tartaj et al. (2003) reported that the size, shape, and magnetic dipole moment of the particle also play a role in in vivo experiments. Properties of nanoparticles like longer sedimentation times, higher surfaces areas, and smaller magnetic dipole-dipole interactions might facilitate their use. Nevertheless, a study of the adverse effects toward their environment is necessary for every specific application.

\section{Conclusions}

Oleic acid-coated nanoparticles were functionalized by a reaction with trialkoxy silanes. The reaction takes place in an ultrasonication bath, which reduces the reaction time and prevents crosslinking. The successful coating procedure of the nanoparticles' surface was proven by FTIR measurements. Multiple techniques (TEM, XRD, VSM) proved the composition of the magnetic core. The obtained functionalized nanoparticles can be dispersed in various aqueous environments including human serum and plasma. Their stability under these conditions was addressed by zeta potential and absorbance measurements, showing a strong relation between the colloidal stability and the $\mathrm{pH}$ of the solution. Although PEG-coated nanoparticles also exhibit this dependency, steric hindrance is expected to be more prominent here. In general, the generic method described here allows the introduction of various functional groups on the surface of the nanoparticles. This is particularly useful for subsequent coupling reactions to fluorescent probes, proteins, or substrates. Therefore, we believe that this type of superparamagnetic nanoparticle can be of major importance for future research and applications in biomedicine.

Acknowledgments We are grateful to Bart Goderis for help with the XRD measurements, to Johan Billen for conducting the TEM measurements, and to Marlies Van de Wouwer for preparing the serum and plasma. We also express our thanks to S. Vandendriessche, M.K. Vanbel, and V.K. Valev for the useful and inspiring discussions. This work was financially supported by Grant G.0618.11 N of the Fund for scientific research, Flanders (FWO-V); the Agency for Innovation by Science and Technology in Flanders (IWT), and the KU Leuven (GOA). M.B. and W.B. are grateful for support from the IWT.

Open Access This article is distributed under the terms of the Creative Commons Attribution License which permits any use, distribution, and reproduction in any medium, provided the original author(s) and the source are credited.

\section{References}

Barreto JA, Matterna M, Graham B, Stephan H, Spiccia L (2011) Synthesis, colloidal stability and $64 \mathrm{Cu}$ labeling of 
iron oxide nanoparticles bearing different macrocyclic ligands. N J Chem 35:2705-2712. doi:10.1039/c1nj20558g

Bhattacharya D, Sahu SK, Banerjee I et al (2011) Synthesis, characterization, and in vitro biological evaluation of highly stable diversely functionalized superparamagnetic iron oxide nanoparticles. J Nanoparticle Res 13:41734188. doi:10.1007/s11051-011-0362-7

Bronstein LM, Huang X, Retrum J, Schmucker A, Pink M, Stein BD, Dragnea B (2007) Influence of iron oleate complex structure on iron oxide nanoparticle formation. Chem Mater 19:3624-3632. doi:10.1021/cm062948j

Brullot W, Valev VK, Verbiest T (2011) Magnetic-plasmonic nanoparticles for the life sciences: calculated optical properties of hybrid structures. Nanomedicine 8(5):559-568. doi:10.1016/j.nano.2011.09.004

Brullot W, Reddy NK, Wouters J, Valev VK, Goderis B, Vermant J, Verbiest T (2012) Versatile ferrofluids based on polyethylene glycol coated iron oxide nanoparticles. J Magn Magn Mater 11:1919-1925. doi:10.1016/j.jmmm. 2012.01.032

Can K, Ozmen M, Ersoz M (2009) Immobilization of albumin on aminosilane modified superparamagnetic magnetite nanoparticles and its characterization. Colloids Surf B, Biointerfaces 71:154-159. doi:10.1016/j.colsurfb.2009. 01.021

Chen ZP, Xu RZ, Zhang Y, Gu N (2008a) Effects of proteins from culture medium on surface property of silanes-functionalized magnetic nanoparticles. Nanoscale Res Lett 4:204-209. doi:10.1007/s11671-008-9226-1

Chen ZP, Zhang Y, Xu K, Xu RZ, Liu JW, Gu N (2008b) Stability of hydrophilic magnetic nanoparticles under biologically relevant conditions. J Nanoscience Nanotechnol 8:6260-6265. doi:10.1166/jnn.2008.343

Cornell RM, Schwertmann U (2003) The iron oxides. Wiley, Weinheim

De Palma R, Peeters S, Van Bael MJ, Van den Rul H, Bonroy K, Laureyn W, Mullens J, Borghs G, Maes G (2007) Silane ligand exchange to make hydrophobic superparamagnetic nanoparticles water-dispersible. Chem Mater 19:18211831

Fadeev AY, McCarthy TJ (2000) Self-assembly is not the only reaction possible between alkyltrichlorosilanes and surfaces: monomolecular and oligomeric covalently attached layers of dichloro- and trichloroalkylsilanes on silicon. Langmuir 16:7268-7274

Fauconnier N, Pons J, Roger J, Bee A (1997) Thiolation of maghemite nanoparticles by dimercaptosuccinic acid. J Colloid Interface Sci 194:427-433

Harder P, Grunze M, Dahint R, Whitesides G, Laibinis P (1998) Molecular conformation in oligo (ethylene glycol)-terminated self-assembled monolayers on gold and silver surfaces determines their ability to resist protein adsorption. J Phys Chem B 102:426-436

Janib SM, Moses AS, MacKay JA (2010) Imaging and drug delivery using theranostic nanoparticles. Adv Drug Del Rev 62:1052-1063. doi:10.1016/j.addr.2010.08.004

Kohler N, Fryxell GE, Zhang M (2004) A bifunctional poly (ethylene glycol) silane immobilized on metallic oxidebased nanoparticles for conjugation with cell targeting agents. J Am Chem Soc 126:7206-7211. doi:10.1021/ ja049195r
Kreuzer HJ, Wang RLC, Grunze M (2003) Hydroxide ion adsorption on self-assembled monolayers. J Am Chem Soc 125:8384-8389. doi:10.1021/ja0350839

Larsen EKU, Nielsen T, Wittenborn T, Birkedal H, VorupJensen $\mathrm{T}$ et al (2009) Size-dependent accumulation of PEGylated silane-coated magnetic iron oxide nanoparticles in murine tumors. ACS Nano 3:1947-1951. doi: 10.1021/nn900330m

Laurent S, Forge D, Port M, Roch A, Robic C, Vander Elst L, Muller RN (2008) Magnetic iron oxide nanoparticles: synthesis, stabilization, vectorization, physicochemical characterizations, and biological applications. Chem Rev 108:2064-2110. doi:10.1021/cr900197g

Ma M, Zhang Y, Yu W, Shen H, Zhang H, Gu N (2003) Preparation and characterization of magnetite nanoparticles coated by amino silane. Colloids Surf A: Physicochem Eng Asp 212:219-226. doi:10.1016/S0927-7757(02)00305-9

Mahmoudi M, Laurent S, Shokrgozar MA, Hosseinkhani M (2011) Toxicity evaluations of superparamagnetic iron oxide nanoparticles: cell "vision" versus physicochemical properties of nanoparticles. ACS Nano 5:7263-7276. doi: $10.1021 / \mathrm{nn} 2021088$

Mason T, Lorimer J (2002) Applied sonochemistry: uses of power ultrasound in chemistry and processing. Wiley, Weinheim

Morel A-L, Nikitenko SI, Gionnet K, Wattiaux A et al (2008) Sonochemical approach to the synthesis of $\mathrm{Fe}(3) \mathrm{O}(4) @$ $\mathrm{SiO}(2)$ core-shell nanoparticles with tunable properties. ACS Nano 2:847-856. doi:10.1021/nn800091q

Park J, An K, Hwang Y, Park JG, Noh HJ, Kim JY, Park JH, Hwang NM, Hyeon T (2004) Ultra-large-scale syntheses of monodisperse nanocrystals. Nat Mater 3:891-895. doi: 10.1038/nmat 1251

Park JW, Bae KH, Kim C, Park TG (2011) Clustered magnetite nanocrystals cross-linked with PEI for efficient siRNA delivery. Biomacromolecules 12:457-465. doi:10.1021/ bm $101244 \mathrm{j}$

Prasad NK, Rathinasamy K, Panda D, Bahadur D (2007) Mechanism of cell death induced by magnetic hyperthermia with nanoparticles of $\gamma-\mathrm{MnxFe}_{2}-\mathrm{xO}_{3}$ synthesized by a single step process. J Mater Chem 17:5042-5051. doi: 10.1039/b708156a

Schweiss R, Welzel PB, Werner C, Knoll W (2001) Dissociation of surface functional groups and preferential adsorption of ions on self-assembled monolayers assessed by streaming potential and streaming current measurements. Langmuir 17:4304-4311

Soderholm K-JM, Shang S-W (1993) Molecular orientation of silane at the surface of colloidal silica. J Dental Res 72:1050-1054. doi:10.1177/00220345930720061001

Speliotis D (1999) Magnetic recording beyond the first 100 years. J Magn Magn Mater 193:29-35. doi:10.1016/ S0304-8853(98)00399-0

Sun S, Zeng H, Robinson DB, Raoux S, Rice PM, Wang SX, Li G (2004) Monodisperse MFe2O4 (M=Fe, Co., Mn) nanoparticles. J Am Chem Soc 126:273-279. doi:10.1021/ja0380852

Sun J, Zhou S, Hou P, Yang Y (2006) Synthesis and characterization of biocompatible $\mathrm{Fe}_{3} \mathrm{O}_{4}$ nanoparticles. J Biomed Mater Res, pp 333-341. doi: 10.1002/jbm.a

Tartaj P, Morales MDP, Veintemillas-Verdaguer S, GonzalezCarreno T, Serna CJ (2003) The preparation of magnetic 
nanoparticles for applications in biomedicine. J Phys D Appl Phys 36:182-197. doi:10.1088/0022-3727/36/13/202

Valiokas R, Svedhem S, Svensson S (1999) Self-assembled monolayers of oligo (ethylene glycol)-terminated and amide group containing alkanethiolates on gold. Langmuir 15:3390-3394

Wang Y, Wong JF, Teng X, Lin XZ, Yang H (2003) "Pulling" nanoparticles into water: phase transfer of oleic acid stabilized monodisperse nanoparticles into aqueous solutions of $\alpha$-cyclodextrin. Nano Lett 3:1555-1559. doi:10.1021/ $\mathrm{nl034731 \textrm {j }}$
Xu R (2001) Particle characterization: light scattering methods. Kluwer Academic Publishers, Greenbelt, p 403

Zhang Y, Kohler N, Zhang M (2002) Surface modification of superparamagnetic magnetite nanoparticles and their intracellular uptake. Biomaterials 23:1553-1561

Zhang L, He R, Gu H (2006) Oleic acid coating on the monodisperse magnetite nanoparticles. Appl Surf Sci 253:26112617. doi:10.1016/j.apsusc.2006.05.023 